\title{
Sweet dreams and gut feelings: endocrinology makes new connections
}

\author{
Aart Jan van der Lely
}

Until some years ago, endocrinology was in danger of becoming dull. One of the reasons was that the field confined itself to studying only what endocrine glands produced; many times endocrinology was just a matter of describing 'hyper-hormonemia' or 'hypo-hormonemia'. The progress that was made in the knowledge of signal transduction in other fields of medical science, such as immunology and hemato-oncology, was much more impressive than the progression that could be observed in classical endocrinology.

Much to our pleasant surprise, things have changed dramatically. As an example, our knowledge about the role of G-protein-coupled receptors in endocrinology has made tremendous strides. Impressive progress has also been made, for example, with regard to the development of new treatment modalities for diabetes. The understanding that, firstly, gut hormones can modify insulin secretion and sensitivity, and that, secondly, the central nervous system can do the same thing made this progress possible. This jump forward is not that surprising now that we endocrinologists have come to realize that there is much in common between the way the nervous system controls the gut and way it controls the pituitary. Of course, the brain uses the pituitary gland to control, for example, growth, adrenal function, thyroid function, and reproduction, but many of the hormones involved in this control have clear effects on metabolism as well.

At a further anatomical distance from the brain, the nervous system also controls metabolism by modifying insulin release both directly and indirectly; the latter by the regulation of the secretion of other hormones such as glucagon-like peptides, peptide $Y Y$, and ghrelin, just to mention a few. In exchange for all this, afferent signals from the gut also reach

\section{There is much \\ in common \\ between \\ the way the \\ nervous \\ system \\ controls the \\ gut and way \\ it controls the pituitary}

AJ van der Lely is Chief and Professor of Clinical Endocrinology at the Department of Medicine, Section of Endocrinology and Head of the Clinical Research Unit at the Erasmus University Medical Center, Rotterdam, The Netherlands.

\section{Competing interests}

The author declared he has no competing interests.

www.nature.com/clinicalpractice doi:10.1038/ncpendmet0188 the brain and the hypothalamic areas to inform them about what and how much food has been consumed, whether or not to have an appetite, and whether to make the body more or less insulin-resistant. Most of the time we do not think of the pituitary as part of the gastrointestinal system, but, from an ontogenic point of view, the anterior pituitary in fact still is.

Communication between the hypothalamus and the pituitary reveals a remarkable degree of complexity, and there are striking similarities between this interplay and the crosstalk between the central nervous system and parts of the gastrointestinal system. Recent data indicate that the sympathetic and parasympathetic nervous systems show a delicate interplay with most intra-abdominal organs, including the liver, pancreas, and intra-abdominal fat. On top of that, higher brain functions such as sleep (especially deep, delta-wave sleep) have been connected with issues such as insulin sensitivity and obesity; moreover, some stages of sleep—and particularly sleep quality-seem to be determinants of anterior pituitary function. An example is the delta-dependent release of growth hormone. Indeed, things have become very complicated but this opens many more avenues for exploration.

Modern biochemistry enables the design of agonists and antagonists to many of the target receptors that are believed to have a pivotal role in metabolic control. The same is true for the development of better sleep-promoting drugs. Nowadays, it is mainly improving quality of sleep rather than increasing sleep duration that is the aim of this type of drug research. So, endocrinology has entered a new era that promises potential solutions for at least two of the largest medical problems that society encounters: diabetes and obesity. Today, endocrinology is fun again. 